\title{
Correlation between serum and salivary house dust mite specific immunoglobulins in Allergic Rhinitis (AR) patients and non-allergic rhinitis matched controls
}

\begin{abstract}
Background and objectives: Allergic rhinitis (AR) is characterised by inflammation of the nasal mucosa associated with IgE mediated immune response to specific allergens. Studies have shown that the most commonly implicated aeroallergens in AR are house dust mites. Dermatophagoides pteronyssinus (Dp), Dermatophagoides farinae (Df) and Blomia tropicalis $(\mathrm{Bt})$ are the three most common mite species in Malaysia. Previous studies have reported the presence of high $\operatorname{IgE}$ and non- $\operatorname{IgE}$ antibodies in both serum and saliva samples of AR patients, but the correlation between them is not clear. The objective of this study was to determine the levels and correlation of mite specific $\operatorname{IgE}$ and $\operatorname{IgA}$ against $D$. farinae and $B$. tropicalis in serum and salivary samples of AR patients and nonallergic rhinitis matched controls.

Methods: A total of 205 adults were studied, they included 103 with AR and 102 healthy controls matched for age and gender. Among the 103 AR patients, 20 had concomitant asthma. Indirect ELISA was used to measure the levels of serum and salivary $\operatorname{IgE}$ and $\operatorname{IgA}$ respectively.

Results: Correlation between the serum and salivary antibody levels was analysed using Spearman correlation test, while the differences between groups were analysed using MannWhitney test and Kruskal Wallis test. Serum IgE and IgA levels against D. farinae and $B$. tropicalis were higher in AR patients. Salivary IgA against D. farinae and salivary IgE against $B$. tropicalis were significantly higher in AR patients. Serum and salivary IgE levels were positively correlated with serum and salivary IgA levels in AR subjects against both mite species. There was no significant difference in antibodies levels between asthmatic AR and non-asthmatic AR to both mite species in serum and saliva samples. Antibodies levels were not correlated with severity of signs and symptoms in AR patients.
\end{abstract}

Conclusion: The presence of high IgA level in saliva might be a potential investigation tool to test for allergic rhinitis when blood sampling is not an option.

Keyword: $\quad$ Allergic rhinitis; Dermatophagoides pteronyssinu; Dermatophagoides farina; Blomia tropicalis ; Serum immunoglobulins; Salivary immunoglobulins 